\title{
EDITORIAL
}

\section{Step Care Management of Heart Failure}

University Heart Journal 2017; 13(1): 1

Heart failure (HF) is the end result of various cardiac and non-cardiac conditions of diverse aetiologies. With the improvement of care of acute coronary syndrome with drugs and coronary intervention the mortality rate is gradually decreasing. However with the aging population the incidence of heart failure syndrome is gradually increasing. Few newer drugs and devices have been approved for the improved management of heart failure which has proved to reduce mortality in addition to the already available evidence based drugs.

The severity of HF is assessed clinically by NYHA Grade and objectively by echocardiographic measurement of left ventricular ejection fraction (LVEF). Treatment of heart failure is guided by NYHA class \& LVEF. The aim of the treatment is to improve the functional class and reduce mortality. With judicious use of the currently available drugs and devices in a step care fashion it is expected to improve prognosis of HF. In the following discussion we have outlined in brief the various drugs and devices which have been advocated in step care management of heart failure.

The time tested ACE inhibitors (ACEI) can be used in all NYHA class. Introduce ACEI only if $\mathrm{Cr}<221 \mathrm{mcmol} / \mathrm{L}$ or $<2.5 \mathrm{mg} / \mathrm{dl}$ or eGFR $>30 \mathrm{ml} / \mathrm{min} / 1.73 \mathrm{~m}^{2}$ and a normal $\mathrm{K}^{+}$. Following treatment If creatinine rises to 265-310 $\mathrm{mcmol} / \mathrm{L}$ or $\mathrm{K}>5.5$, ACEI should be halved (ESC). Isosorbid dinitrate- hydralazine combination (H-ISDN) can be used if ACEI is intolerated or if tolerated with residual symptoms (NYHA II-IV) \& EF $>35$. Betablocker is introduced early (NYHA II-III). Ivabradine is added in $\mathrm{HF}$ if $\mathrm{EF}<35 \%$ and $\mathrm{HR}>70 / \mathrm{min}$ despite having beta-blockers or patient is intolerant to beta-blocker. Ivabradine is ineffective in atrial fibrillation. Spironolactone is added if NYHA $>$ III with EF $<35$. Eplerenone is indicated post MI if EF $<40$. Sacubitril/ valsartan combination (ARNi) is used in patients with NYHA II-IV with LVEF $\leq 35 \%$ who are already using ACEI.

CRT-D is indicated in patients with NYHA II, QRS> $130 \mathrm{~ms}$, LBBB morphology and $\mathrm{EF}<30$. In patients with NYHA III-IV, QRS $>120 \mathrm{~ms}$ (LBBB morphology), EF $<35$ then CRT-P/D is indicated. (ESC). The National institute of Health and Care Excellence (NICE) guideline requirements' for CRT implantation slightly differs with ESC guidelines. According to NICE, in patients with ischaemic cardiomyopathy with NYHA III-IV, and EF $<35$, CRT-D is indicated. If QRS $>150 \mathrm{~ms}$ or if QRS $120-$ $150 \mathrm{~ms}$, then presence of left ventricular dyssynchrony is required in echocardiography is required before CRTD implantation.

\author{
Dr Manzoor Mahmood \\ Associate Professor of Cardiology, Bangabandhu \\ Sheikh Mujib Medical University (BSMMU), Dhaka \\ Professor Harisul Hoque \\ Department of Cardiology, BSMMU, Dhaka
}

